\title{
EXPERIMENTS ON THE PRODUCTION AND MEASUREMENT OF HIGH VACUA.
}

\author{
By J. W. Woodrow.
}

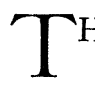

HIS article describes the actual construction of a sensitive Knudsen absolute manometer and explains its use for the measurement of low pressures. An account is also given of some methods employed for obtaining these pressures.

\section{INTRODUCTION.}

The theory of an absolute manometer, which is independent of the gas to be measured, has been given by Knudsen. ${ }^{1}$ However he merely gives data to show that his theory is confirmed by experiment and does not describe the actual construction of such a gauge. A brief outline of the operation of one type of the Knudsen manometer has been given by Angerer, ${ }^{2}$ but some difficulty was experienced by the author of this article in trying to make a sensitive gauge according to his instructions. Therefore, as quite a little time has been spent in this laboratory on the construction of a very sensitive gauge which would be at the same time easily made and simple in operation, it was thought that the results of these experiments were of sufficient value to warrant publication.

Previous experiments had shown that for the measurement of very low pressures the whole gauge must be so constructed that it could be placed in an oven and heated to a temperature of about $200^{\circ} \mathrm{C}$. while on the pump. It was further desired to make the volume of the gauge as small as possible and to eliminate all metal which was not absolutely necessary. The gauge, described by Angerer, could not be heated without danger of cracking, a difficulty which has been overcome in the gauge to be described below.

\section{Description of Apparatus.}

Several different gauges were constructed varying in sensitivity so as to be used at different pressures. A typical gauge is shown in Figs. I and 2 and the electrical circuits are given in Fig. 3. The glass rods $G G$ served as supports for the metallic parts of the gauge. All the internal electrical connections and adjustments, with the exception of the final leveling, were made before the outer glass wall $O O$ was sealed on at $S S$.

${ }^{1}$ M. Knudsen, Ann. d. Phys., 32, p. 809, I9ro.

2 E. v. Angerer, Ann. d. Phys., 4I, p. Io, I9r3. 
The suspension $W$ was a phosphor-bronze ribbon $50 \mathrm{~mm}$. in length which had been obtained from W. G. Pye \& Co. and was listed by them as No. oooo. The movable vane $V V$ consisted of a rectangular frame of aluminum $0.076 \mathrm{~mm}$. in thickness, the dimensions of the outer rectangle

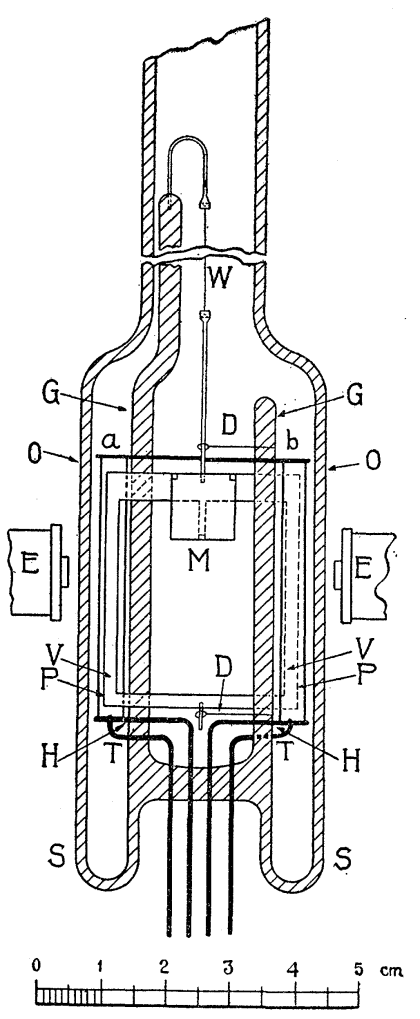

Fig. 1. being $30 \times 36 \mathrm{~mm}$. and of the inner 26 $\times 30 \mathrm{~mm}$. The heating plates $P P$ were platinum strips $4 \mathrm{~mm}$. wide, $40 \mathrm{~mm}$. long and $0.025 \mathrm{~mm}$. thick. The deflections of the movable vane were obtained in the usual way by the reflection of a beam of light from the mirror $M$. Fig. 2 is a crosssectional view through the middle of Fig. I.

All of the platinum connections were made by electric welding, as that was found much more satisfactory than the use of any kind of solder, especially when heated. After a little practice, it was possible to weld the thin platinum heating vanes to the heavy platinum wire so as to make a perfectly continuous contact throughout its width. The phosphor-bronze suspension was connected at both ends by threading through three small holes drilled into the flattened extremities of the platinum and aluminum wires respectively. The small loops $D D$ were so placed that they supported the movable vane $V$ except when the gauge was leveled for taking readings. This made the gauge readily portable and, by placing in the inverted position when connected to the molecular pump, the danger of the breaking of the suspension by vibration was eliminated. One gauge of medium sensitivity was constructed so as to be sufficiently steady to be used when connected directly to the molecular pump. Large glass tubing was employed in all the connecting portions of the apparatus.

The movable vane used by Angerer consisted of silvered mica, but the present investigation has shown that such a vane is very susceptible to variations in the light falling upon it. An ordinary forty-watt tungsten lamp placed $50 \mathrm{~cm}$. away so as to shine on the vane would cause a deflection of $100 \mathrm{~mm}$. when the pressure was about $10^{-5} \mathrm{~mm}$. of $\mathrm{Hg}$. This phenomenon was shown not to be a direct heat effect by the fact that a 
non-luminous Bunsen flame had no effect when brought quite close to the apparatus and that with a luminous flame the effect was immediately observed. When the silvered-mica was replaced by aluminum, the effect of light variations was small enough to be neglected. The moving system was electrically connected through the suspension to that terminal of the heating strips which was grounded, the whole being screened from external electrical disturbances by an earthed silver coating on the outside of the glass walls. In an earlier experiment, the silvering was on the inside of the walls, but this was unsatisfactory as it was impossible to free the coating from gases while connected to the molecular pump. However the above method of electrical screening was sufficiently good so long as there was no source of ionization within the apparatus; but if the gauge was connected to an ionization chamber, the electrical dis-

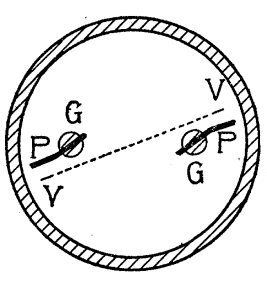

Fig. 2. turbances became very troublesome when the ionization was large. No attempt was made in this case to prevent the diffusion of the ions, although this could be easily accomplished by a suitable wire gauge.

A small electromagnet, shown at $E$ in Fig. I, was employed in bringing the moving vane to rest. This was found to be quite necessary in working with the most sensitive gauges, since in a very good vacuum the damping is so small that the vane will not settle down sufficiently for the taking of readings for some time after an accidental disturbance has set it vibrating. It should be noted that the electromagnet must have either an air core

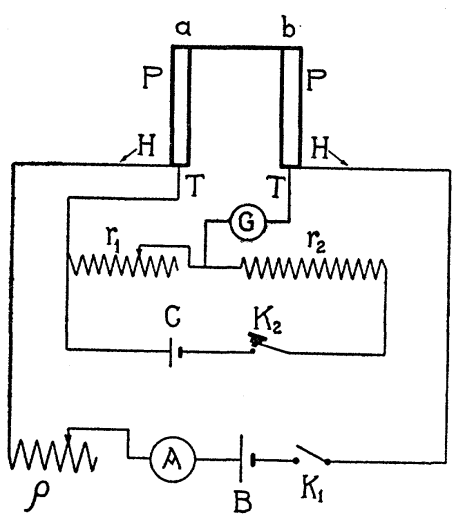

Fig. 3. or one of good, soft Norway iron, for otherwise the residual magnetism will produce a false zero if the aluminum vane is at all magnetic, as was the case with the samples of metal investigated in this laboratory. Under these conditions it is obvious that the electromagnet should be used only for damping and that the exciting current should be shut off while making observations.

Several methods were tried for determining the temperature of the heating strips and that shown in diagram in Fig. 3 was finally settled upon as giving the most satisfactory results. The potentiometer leads $T T$ were connected by electric welding to the very extremities of the platinum heating vanes $P P$. The heating current was regulated by the 
variable resistance $\rho$ and its value was read on the ammeter $A$. The resistance $r_{2}$ was kept constant at I0,000 ohms and $r_{1}$ varied to obtain a balance of the sensitive galvanometer $G$. The potentiometer battery $C$ consisted of a carefully calibrated Weston Standard Cell. This arrangement gave an accurate method of measuring the resistance of the platinum strips $P P$, plus the heavy platinum wire $a b$, the total cold resistance being o.I $7 \mathrm{ohm}$. This cold resistance was determined by plotting the curve connecting resistance and heating current under a constant low pressure and extrapolating backward to the intersection with the axis of resistance. If the resistance is measured for small currents, the value at zero current, that is the cold resistance, can be determined very accurately. A typical curve is shown in Fig. 4. The temperature coefficient of resistance

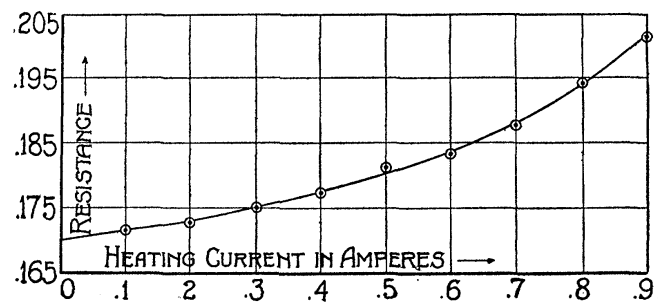

Fig. 4.

of the platinum, which contained a small amount of iridium, was carefully determined and was found to give a linear relation within the range of temperatures employed. The value of the coefficient was $2.35 \times 10^{-3}$ ohms per degree Centigrade. With this system one can determine the mean temperature of the heating strips with sufficient accuracy, the error for temperature differences of about $50^{\circ} \mathrm{C}$. being less than four per cent. It should be mentioned that numerous attempts were made to employ fine thermo-couples, but it was practically impossible to make a good thermal contact with the heaters such that it would remain constant upon heating to $200^{\circ} \mathrm{C}$.

\section{Calculation of the Constants of the Gauge.}

According to the theory given by Knudsen, the gas pressure within the gauge is given by the formula

$$
P=\frac{2 K}{\sqrt{T_{1} / T_{2}}-\mathbf{I}},
$$

where $K$ is the uniform pressure in dynes on each $\mathrm{cm} .^{2}$ of the movable vane and $T_{1}$ and $T_{2}$ are the absolute temperatures of the heating strips and of the moving vane respectively. If the difference in temperature is 
small as compared to the absolute temperature, the formula may be written

$$
P=4 K \frac{T_{2}}{T_{1}-T_{2}} .
$$

It is to be noted that this formula is independent of the nature of the gas or vapor to be measured. The constant $K$ was found in terms of deflection, the constants of the suspension being determined in the usual way from the period of the moving system and the calculated moment of inertia of the suspended rectangular frame. For the gauge whose dimensions are given above, the period of a complete oscillation was Io.o sec. and the calculated moment of inertia of the moving vane was $0.074 \mathrm{gm}$. $\mathrm{cm} .^{2} \quad$ This gives for the pressure

$$
P=2.9 \times \mathrm{IO}^{-5} \frac{T_{2}}{T_{1}-T_{2}} d \text { dynes, }
$$

or

$$
P=2.2 \times \operatorname{IO}^{-8} \frac{T_{2}}{T_{1}-T_{2}} d \mathrm{~mm} . \text { of } \mathrm{Hg} .,
$$

where $d$ is the deflection in $\mathrm{mm}$. on a scale at a distance of one meter from the mirror. As it is easily possible to obtain a temperature difference of $100^{\circ} \mathrm{C}$. and since a deflection of $0.5 \mathrm{~mm}$. can be observed, the gauge will measure a pressure as low as $3 \times 1^{-8} \mathrm{~mm}$. of $\mathrm{Hg}$.

It was thought that at these very low gas pressures the radiation pressure might have an appreciable effect as compared to that of the molecular bombardment. To test for such an effect, the deflection was obtained for several different values of the temperature of the hot platinum strips, when a very constant pressure had been obtained. If the radiation pressure is small enough to be negligible there ought to be a linear relation between the deflection and the temperature difference, while if it is not negligible the deflection should increase more rapidly. The accompanying table shows that the radiation pressure does not have an appreciable effect at a gas pressure of about $\mathrm{IO}^{-6} \mathrm{~mm}$. of $\mathrm{Hg}$. for temperature differences up to $85^{\circ} \mathrm{C}$.

TABLE I.

\begin{tabular}{c|c|c}
\hline Temp. Diff. in ${ }^{\circ} \mathrm{C}$. & Deflection in Mm. & $\begin{array}{c}\text { Calculated Pressure } \\
\times \mathbf{1 0}^{-6} \mathbf{M m} \text {. Hg. }\end{array}$ \\
\hline 8 & 4 & 3.4 \\
20 & 9 & 3.1 \\
35 & 17 & 3.3 \\
65 & 28 & 2.7 \\
83 & 40 & 3.2 \\
\hline
\end{tabular}




\section{Measurements of Low Pressure.}

The lowest pressure obtained with a Gaede molecular pump was measured by a specially constructed gauge, the pressure being $3 \times \mathrm{IO}^{-5}$ $\mathrm{mm}$. of $\mathrm{Hg}$. A Gaede rotary-box pump was used as a fore-pump giving a pressure of o.or $\mathrm{mm}$. of $\mathrm{Hg}$., and the molecular pump was run at 8,000 R.P.M. The above vacuum was obtained after pumping for about 3 hours and with the gauge connected as closely as possible to the molecular pump, the total volume consisting only of the gauge and connecting glass-tubing. However in this case the glass walls of the gauge were not heated.

It is interesting to compare the vacuum given by the molecular pump with that obtained when a side tube containing cocoanut charcoal is immersed in liquid air. By the latter method the pressure was reduced after about 36 hours to $2 \times \mathrm{IO}^{-7} \mathrm{~mm}$. of $\mathrm{Hg}$. If the liquid air were removed, the pressure would increase to a value somewhat higher than that measured when the gauge was sealed off the molecular pump, but it would then decrease again, slowly approaching the value obtained at sealing off. On the other hand if the liquid air is replaced before this gradual decrease begins the vacuum very quickly reaches the value previously obtained after about 36 hours. This effect is probably due to absorption on the walls of the glass and on the metallic parts of the apparatus which makes the original action of the charcoal and liquid air so slow. If the walls are carefully heated while the gauge is connected to the molecular pump, this phenomenon is not so pronounced.

The lowest pressure which we have measured was obtained by the use of charcoal and liquid air in the usual way supplemented by the evacuating action of a tungsten filament sealed into a side tube and kept running for 130 hours. The pressure had reached the value $5 \times \mathrm{IO}^{-8}$ $\mathrm{mm}$. of $\mathrm{Hg}$. when the investigation was stopped by an accident to the apparatus.

Some interesting data was obtained on the efficacy of rubber valvegrease for holding a good vacuum. A system, which contained three large stopcocks and a large ground-glass joint, attained a vacuum of $\mathrm{IO}^{-6} \mathrm{~mm}$. of $\mathrm{Hg}$. under the action of charcoal and liquid air. The vacuum remained constant at this value for several days while the charcoal was kept immersed in the liquid air. However when the charcoal tube was disconnected by closing one of the stopcocks, the pressure gradually increased but at a very slow rate.

As the Knudsen absolute manometer measures the pressure independently of the nature of the gas, it affords a convenient method of determining the rate at which charcoal immersed in liquid air will remove 
different gases. This effect was investigated with oxygen, nitrogen and hydrogen, but it was impossible to get an accurate quantitative comparison as the oxygen was removed too quickly under the action of the liquid air. However some idea of the relative rates may be obtained from the data in Table II. In each case the pressure before inserting the known gas was reduced to the same value, viz. $\mathrm{I} .5 \times 1 \mathrm{IO}^{-6} \mathrm{~mm}$. of $\mathrm{Hg}$., the charcoal being thoroughly heated while connected to the molecular pump. Hydrogen was investigated first, then oxygen and nitrogen in the order named. The values given in the first column are the times after

TABLE II.

\begin{tabular}{|c|c|c|c|}
\hline \multirow{2}{*}{ Time of Action. } & \multicolumn{3}{|c|}{ Pressure in $\mathrm{Mm}$. of $\mathrm{Hg}$. for } \\
\hline & Hydrogen. & Oxygen. & Nitrogen. \\
\hline Zero. & $485 \times 10^{-6}$ & $500 \times 10^{-6}$ & $450 \times 10^{-6}$ \\
\hline 5 seconds. & $\underline{-}$ & $290 \times 10^{-6}$ & $\underline{-120}$ \\
\hline 10 seconds. . & - & $170 \times 10^{-6}$ & - \\
\hline 1 minute. ......... & $460 \times 10^{-6}$ & $2 \times 10^{-6}$ & $15 \times 10^{-6}$ \\
\hline 5 minutes. . . . . . . . . & $410 \times 10^{-6}$ & $1.5 \times 10^{-6}$ & \\
\hline 20 minutes. . . . . . . . & $290 \times 10^{-6}$ & & 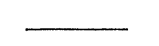 \\
\hline 1 hour. $\ldots \ldots \ldots \ldots \ldots$ & $190 \times 10^{-6}$ & - & $5 \times 10^{-6}$ \\
\hline 3 hours. $\ldots \ldots \ldots \ldots \ldots$ & $135 \times 10^{-6}$ & $1.5 \times 10^{-6}$ & $\underline{\mathrm{n}}$ \\
\hline 10 hours. ............ & $135 \times 10^{-6}$ & $1.5 \times 10^{-6}$ & $5 \times 10^{-6}$ \\
\hline
\end{tabular}

the charcoal tube has been connected to the gauge by opening the stopcock. For the oxygen, the measurements were taken by leaving the stopcock open only for a few seconds at a time. No special care was taken in the preparation of these gases and consequently they were not very free from other gases and vapors but were sufficiently pure to clearly show the phenomenon.

Research Laboratory of The American Telephone and Telegraph Company AND the Western Electric Company,

New YoRk City. 